\title{
Deep sequencing for de novo construction of a marine fish (Sparus aurata) transcriptome database with a large coverage of protein-coding transcripts
}

\author{
Josep A Calduch-Giner ${ }^{1}$, Azucena Bermejo-Nogales', Laura Benedito-Palos ${ }^{1}$, Itziar Estensoro², \\ Gabriel Ballester-Lozano ${ }^{1}$, Ariadna Sitjà-Bobadilla ${ }^{2}$ and Jaume Pérez-Sánchez ${ }^{1 *}$
}

\begin{abstract}
Background: The gilthead sea bream (Sparus aurata) is the main fish species cultured in the Mediterranean area and constitutes an interesting model of research. Nevertheless, transcriptomic and genomic data are still scarce for this highly valuable species. A transcriptome database was constructed by de novo assembly of gilthead sea bream sequences derived from public repositories of mRNA and collections of expressed sequence tags together with new high-quality reads from five cDNA 454 normalized libraries of skeletal muscle (1), intestine (1), head kidney (2) and blood (1).
\end{abstract}

Results: Sequencing of the new 454 normalized libraries produced 2,945,914 high-quality reads and the de novo global assembly yielded 125,263 unique sequences with an average length of $727 \mathrm{nt}$. Blast analysis directed to protein and nucleotide databases annotated 63,880 sequences encoding for 21,384 gene descriptions, that were curated for redundancies and frameshifting at the homopolymer regions of open reading frames, and hosted at http://www.nutrigroup-iats.org/seabreamdb. Among the annotated gene descriptions, 16,177 were mapped in the Ingenuity Pathway Analysis (IPA) database, and 10,899 were eligible for functional analysis with a representation in 341 out of 372 IPA canonical pathways. The high representation of randomly selected stickleback transcripts by Blast search in the nucleotide gilthead sea bream database evidenced its high coverage of protein-coding transcripts.

Conclusions: The newly assembled gilthead sea bream transcriptome represents a progress in genomic resources for this species, as it probably contains more than $75 \%$ of actively transcribed genes, constituting a valuable tool to assist studies on functional genomics and future genome projects.

Keywords: Sparus aurata, Next-generation sequencing, De novo assembly, Transcriptome, Database

\section{Background}

The gilthead sea bream (Sparus aurata) is a member of the Sparidae family widely and successfully cultured in the Mediterranean region. During the last decade more than 1,200 scientific papers have focused on gilthead sea bream nutrition, immune response, physiology and genetics. This high valuable fish for aquaculture industry becomes, thereby, an interesting animal model, and the development of molecular and genomic tools for research is highly desirable. Previous attempts have been

\footnotetext{
* Correspondence: jaime.perez.sanchez@csic.es

${ }^{1}$ Nutrigenomics and Fish Growth Endocrinology Group, Department of Marine Species Biology, Culture and Pathology, Institute of Aquaculture Torre de la Sal, Castellón, CSIC, Spain

Full list of author information is available at the end of the article
}

made in this way and the assembly and annotation of more than 40,000 expressed sequence tags (ESTs) allowed the development of specific gilthead sea bream microarrays that have been used in transcriptomic studies of crowding stress [1] and parasite and nutritional challenges [2,3]. Microarray approaches have also been done in this species to assess transcriptome differences between two early larval stages [4], to underline the liver transcriptomic response after cortisol administration $[5,6]$ or to identify the key genes involved in the re-epithelialization process after scale removal [7]. Nevertheless, genomic tools still remain relatively scarce in gilthead sea bream and need to be further improved in comparison to other cultured fish, such as Atlantic

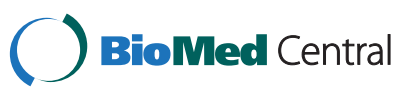


salmon (Salmo salar) [8], common carp (Cyprinus carpio) [9], Nile tilapia (Oreochromis niloticus) [10] or turbot (Scophthalmus maximus) [11], for which large ESTs collections generated by Sanger sequencing of cDNA libraries are currently available.

With the advent of the next generation sequencing technologies, the gathering of large amounts of sequence data for a given organism at affordable costs is more feasible [12], and high-throughput 454 pyrosequencing has been used to explore the transcriptome of rainbow trout (Oncorhynchus mykiss) [13,14], Atlantic cod (Gadus morhua) [15] and turbot [16]. In the case of gilthead sea bream, two deep sequencing studies have been reported from whole larval tissues [17] and skeletal muscle [18], yielding 68,289 and 43,461 assembled sequences, respectively. Nevertheless, the assembly of high-throughput sequences from one unique tissue usually results in relatively short sequences. As the annotation success of a sequencing project is highly dependent on the size of the assembled sequences, approaches conducted to obtain longer sequences become desirable. At this respect, it has been proved in some animal models, including rainbow trout [13,14], that the use of two or more tissues for sequencing and assembly increases the number of annotated genes. On this basis, the primary goal of the present study was to generate a large amount of gilthead sea bream transcriptomic reads from metabolically and immunologically relevant tissues by means of the construction of five 454 pyrosequencing libraries, combining them with Sanger sequences from public repositories and our own published data. It comprised nine previously constructed suppression subtractive hybridization (SSH) libraries that were obtained from a variety of tissues (liver, gills, brain, intestine, head kidney, adipose tissue) from animals exposed to confinement stress, parasite infection or a nutritional stress (essential fatty acid deficiencies) [1-3]. Having this into account, tissues selected for the improvement of the current knowledge on gilthead sea bream transcriptome by means of high-throughput sequencing were related to animal growth (skeletal muscle), nutrient digestion (intestine) and immune response (head kidney at two stages of parasitic infection). Because of the importance of the development of non-lethal diagnostic methods, blood was also considered for 454 sequencing. The second goal of this work was to build a reliable assembly database, with a high confidence of functional annotation by means of similarity searches, gene ontology (GO) terms and detailed insights on pathway analysis for its use as a practical tool by the scientific community. This will assist gene discovery and studies on functional genomics, as well as future genome projects of this important fish species.

\section{Results and discussion}

Five normalized cDNA libraries from skeletal muscle, intestine, blood and head kidney (short and chronic parasite infection) were constructed and sequenced in separate half-runs using 454-Titanium FLX technology. Table 1 summarizes the results for each one of the five normalized 454 libraries. Collectively, more than 2,900,000 high-quality reads averaging 313 nt each were produced, representing $0.9 \mathrm{~Gb}$ of expressed sequence information. For each library, an independent assembly was performed with the Newbler software, and the number of assembled contigs ranged within libraries from 7,800 to 14,008 with an average length of $831-$ $1,148 \mathrm{nt}$. At this stage and regardless of the tissue library, the number of singletons that failed to assemble

Table 1 Statistics for 454 pyrosequencing libraries

\begin{tabular}{|c|c|c|c|c|c|}
\hline & Skeletal muscle & Intestine & Blood & HK-38 & HK-105 \\
\hline \multicolumn{6}{|l|}{ Pyrosequencing reads } \\
\hline High-quality reads & 447,166 & 614,899 & 576,796 & 577,112 & 729,941 \\
\hline Average read length (bp) & 294 & 304 & 290 & 349 & 320 \\
\hline Total Megabases & 131.5 & 186.9 & 167.3 & 201.4 & 233.6 \\
\hline \multicolumn{6}{|l|}{ Assembly statistics } \\
\hline Number of contigs & 7,808 & 9,475 & 12,003 & 14,008 & 12,474 \\
\hline Reads assembled & 314,628 & 482,080 & 389,934 & 392,195 & 508,826 \\
\hline Average contig length (bp) & 968 & 1,090 & 831 & 1,059 & 1,148 \\
\hline Number of singletons & 48,750 & 80,527 & 114,984 & 105,431 & 91,885 \\
\hline Average singleton length (nt) & 316 & 298 & 284 & 318 & 334 \\
\hline Total consensus Megabases & 23.0 & 34.3 & 42.7 & 48.4 & 45.0 \\
\hline Average sequences coverage & 6.4 & 6.3 & 4.0 & 4.2 & 5.8 \\
\hline
\end{tabular}


remained relatively high. As a result of this, the $67-77 \%$ of the information derived from each individual library was supported only by short singleton reads (298$334 \mathrm{nt}$ in length), which is particularly critical given that most assembly software programs include default steps that discard them to give more reliable results, with the unadvised loss of potentially valuable data [19]. This is a complex issue and to solve it, we considered a unique global assembly with the already available mRNA sequences and EST collections in combination with all the unassembled highquality reads derived from the five 454 libraries (Figure 1).

As a result of the above integrative approach, the average number of reads composing each contig (contig depth) increased from 4.0-6.4 in the individual tissue assemblies to 22.2 in the global assembly (Table 2). This large improvement increased the reliability of the transcriptomic database, yielding 113,927 assembled contigs with an average length of 762 nt that cover the 95.3\% of the consensus megabases (86.8 out of 91.1). This finding agrees with the observation that the total number of assembled contigs increased from 43,507 to 50,515 when five skeletal muscle libraries of gilthead sea bream were globally and not individually assembled [18]. Both in that and the present study, the number of 454 reads used for the assembly is of the same order of magnitude (approximately 3 millions), but in our case they are derived from four different tissues and after combination with 82,000 Sanger reads from public repositories the final number of contigs was more than the double $(113,927)$ of that reported in [18].

Of a total number of 125,263 sequences (contigs and singletons), the $51.0 \%$ (63,880 sequences) were annotated using nucleotide and protein reference databases. This annotation ratio is quite higher than those reported in highthroughput sequencing studies conducted in other fish species of interest in aquaculture, such as turbot (44.8\%) [16], blunt snout bream (Megalobrama amblycephala; 40.5\%) [20] or silver carp (Hypophthalmichthys molitrix; 26.9\%) [21]. This annotation improvement can be explained by the high length (762 nt) of the contigs derived from the present study (Figure 2), which is also inferred when comparisons are made with previous studies in the same species, rendering a contig size of 494 nt and 596 nt in length for 454 libraries of skeletal muscle [18] and whole larvae [17], respectively. The contig depth was increased in parallel and the best BlastX hits for the potentially transcribed proteins yielded 21,384 different Swissprot accessions. This finding suggests that our nucleotide database would contain more than the $75 \%$ of the protein coding transcripts of gilthead sea bream, assuming an average size of 25,000-30,000 genes in a fish with a non-recently duplicated genome $[22,23]$.

With independence of a high coverage of proteincoding transcripts, more than 60,000 assemblies still remain without annotation. This relatively high number of unknown sequences could correspond to more

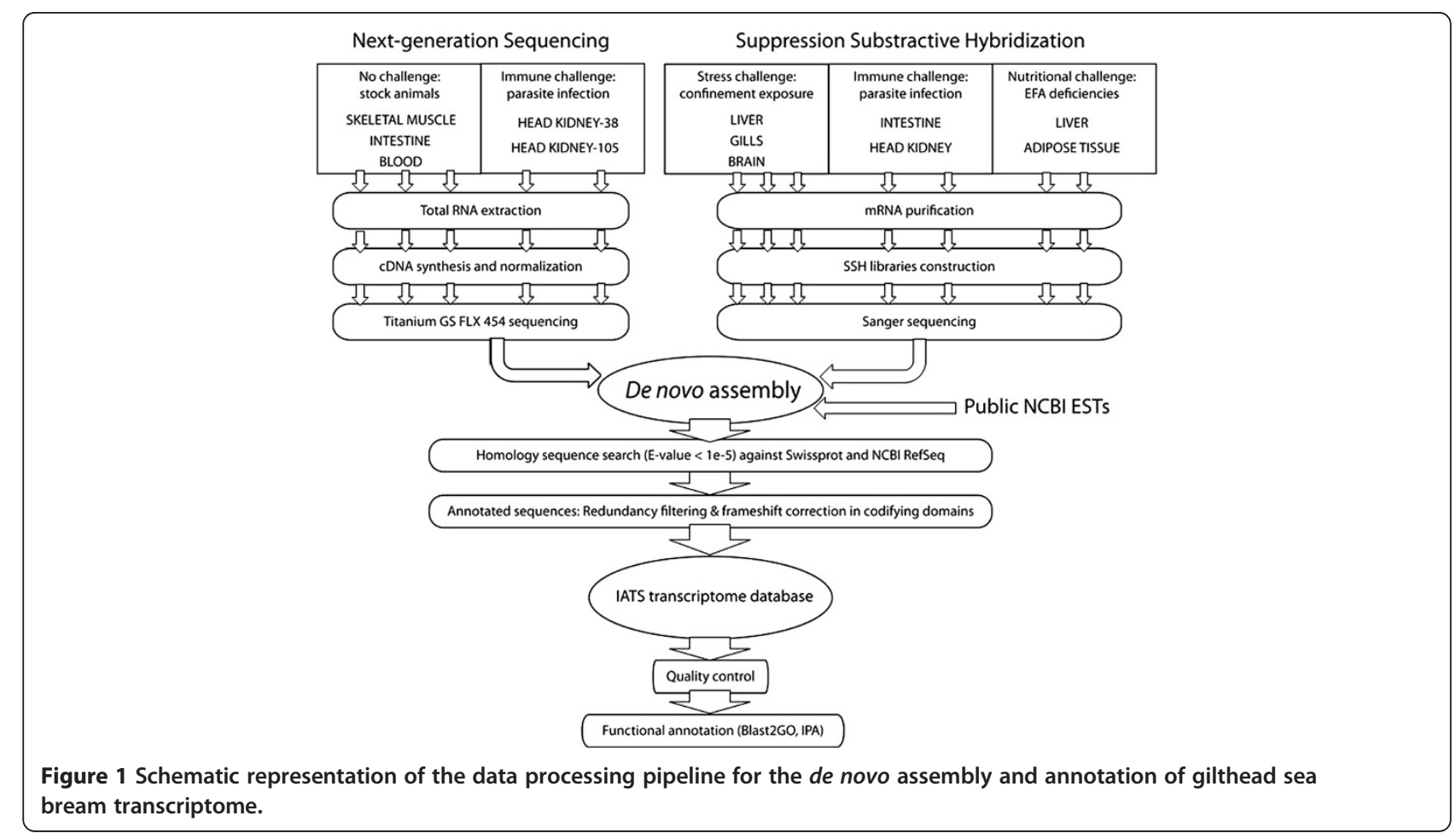


Table 2 De novo assembly statistics

\begin{tabular}{lc}
\hline Assembly statistics & \\
\hline Number of contigs & 113,927 \\
Reads assembled & $2,765,597$ \\
Average contig length (bp) & 762 \\
Number of singletons & 11,336 \\
Average singleton length (nt) & 378 \\
Total consensus Megabases & 91.1 \\
Average sequences coverage & 22.2 \\
\hline
\end{tabular}

divergently evolved genes through vertebrate evolution, though we cannot exclude that some of these sequences may result from assembly artifacts. Alternatively, some of these sequences might correspond to long non-coding RNAs (lncRNAs), which are now emerging as an important class of regulatory transcripts with an extent that increases much more with organism complexity [24,25]. Otherwise, high-throughput sequencing is prone to sequencing errors at homopolymer regions, even when assembled at high coverage, which often give rise to a range of artificial sequences [26-28]. To overcome this issue, an in silico correction step was introduced in the pipeline procedure, which allowed to obtain continuous open reading frames for annotated sequences avoiding frameshifting by edition (insertion or deletion) of single nucleotides at homopolymer regions. With this newly developed tool, up to $34 \%$ of annotated sequences $(21,748$ out of 63,880 assembled sequences) were detected to carry one or more frameshifts. Among them, 21,105 were satisfactorily corrected with the pipeline and only 643 needed a manual curation.

Quality and reliability of the assembled gilthead sea bream database was assessed by BlastX comparison of 200 randomly chosen transcripts of the stickleback transcriptome. Of note, the $71 \%$ of these stickleback transcripts (142 sequences) were originally annotated by the stickleback consortium, and for $94 \%$ of them (133 sequences) a positive result with the same annotation was found using our database as a transcriptome reference (Figure 3A). Besides, reliable annotations were found for 48 sequences out of 58 that were not previously annotated in the stickleback transcriptome (Figure 3B). Again this successful result can be explained by the high average length of the assembled gilthead sea bream sequences, obtained by de novo global assembly of Sanger sequences and high-throughput sequences of four metabolically and immunologically relevant tissues.

Blast2GO analysis of the different annotations (Figure 4) reveals that the most abundant $\mathrm{GO}$ terms related to biological processes were transport (GO:0006810; 2,091 different genes), protein modification process (GO:0036211; 1,558 genes), response to stress (GO:0006950; 1,468 genes), regulation of biological quality (GO:0065008; 1,430 genes) and negative regulation of cellular process (GO:0048523; 1,428 genes). Other highly represented terms are immune system process (GO:0002376; 865 different genes), haemopoiesis (GO:0030097; 289 genes) and coagulation (GO:0050817; 290 genes), which is not surprising given that 3 out of five 454 libraries were derived from blood and head kidney. Regarding skeletal muscle, tissue-specific biological processes like muscle structure development (GO:0061061; 302 genes), muscle cell differentiation (GO:0042692; 200 genes) or muscle contraction (GO:0006936; 156 genes) were also highly represented in the annotated gilthead sea bream transcriptome. Likewise, the intestine participates in nutrient digestion and absorption with also an important role in xenobiotic metabolism [29], and accordingly genes related to the molecular functions hydrolase activity (GO:0016787; 1,821 genes) and oxidoreductase activity (GO:0016491; 637 genes) were highly abundant in our database.

GO terms define gene sets, but the relevance of a given metabolic process or biological pathway in a dataset is determined not only by the number of identified genes, but also by their relative abundance ratio considering the total number of genes involved in each pathway. For this reason, precise mapping of metabolic pathways becomes necessary, and the usefulness of pathway analysis software is largely increasing [30]. To date, the only fish metabolic network available, the public online tool MetaFishNet [31], is still far from completion, so pathway analysis of the gilthead sea bream annotated sequences was performed by means of the Ingenuity Pathway Analysis (IPA) software. Conversion of the annotated gilthead sea bream sequences to IPA elements assumed that the functional classification of genes did not vary too much widely between teleostean fish and model species (human, rat and mouse). In this scenario, from the initial list of 21,384 annotated gilthead sea bream descriptions, the IPA software identified 17,144 Uniprot codes with model species equivalences, and 10,899 of them were eligible for function/pathway analysis. These sequences were represented in 341 IPA canonical pathways mapped from a total of 372, which reinforces the idea that the present gilthead sea bream transcriptome is of high coverage and, thereby, very close to completion of the protein-coding transcriptome. The most representative high-level functional categories are listed in Table 3 with precise information of statistic values and number of represented molecules. The top five canonical pathways were regulation of eIF-4 and p70S6K signaling, purine metabolism, EIF2 signaling, protein ubiquitination pathway, and glucocorticoid receptor signaling. As a corollary, the complete list of canonical pathways with the ratio of gilthead sea bream 


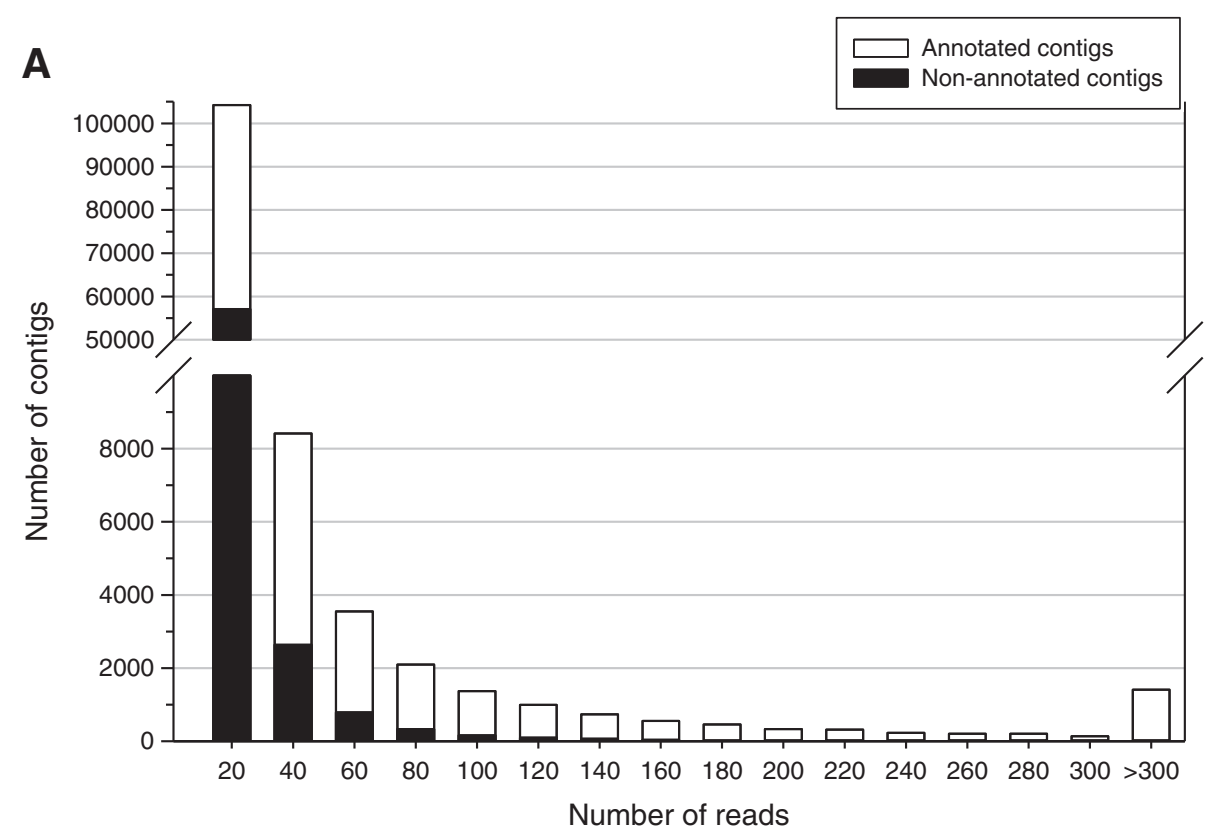

B

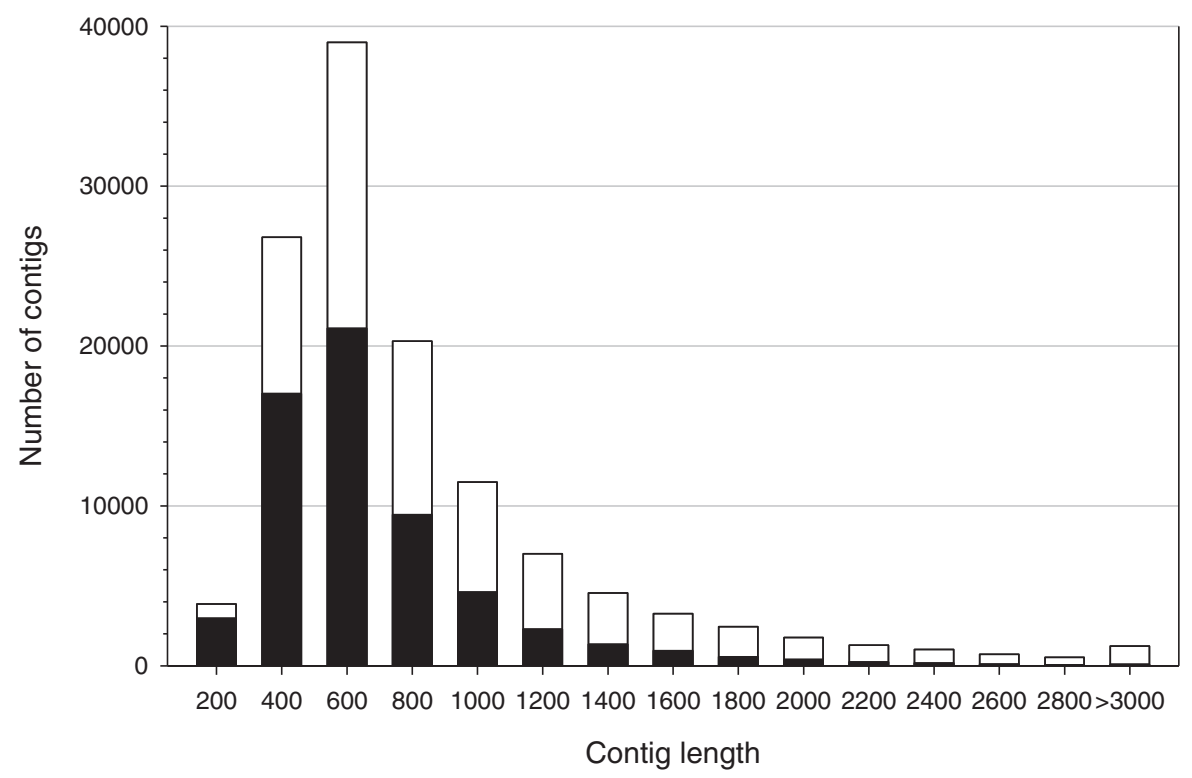

Figure 2 Characteristics of de novo assembled gilthead sea bream transcriptome. (A) Distribution of number of reads per contig. (B) Size distribution of reads after the global assembly. The number of annotated (white bars) and non-annotated sequences (black bars) are represented for each category.

genes present in each one is provided as Additional file 1, and the graphical representation of representative canonical pathways of processes of interest (mTOR signaling, mitochondrial dysfunction, tight junction signaling, B cell receptor signaling, acute phase response signaling and complement system) are presented in Additional file 2.

In order to construct a reliable database of proteincoding transcripts with a minimum of frameshift errors or redundancies, a strict filtering was applied to the annotated sequences after the in silico homopolymer correction step. Sequences with a significant hit against Swissprot were selected, and then sequences encoding for a same annotation regardless of the species were clustered. Filtering selected for each annotation nonoverlaping sequences and putative complete open reading frames, that made a total of 17,809 sequences that were uploaded in our nucleotide database (http://www. nutrigroup-iats.org/seabreamdb). This online resource is 

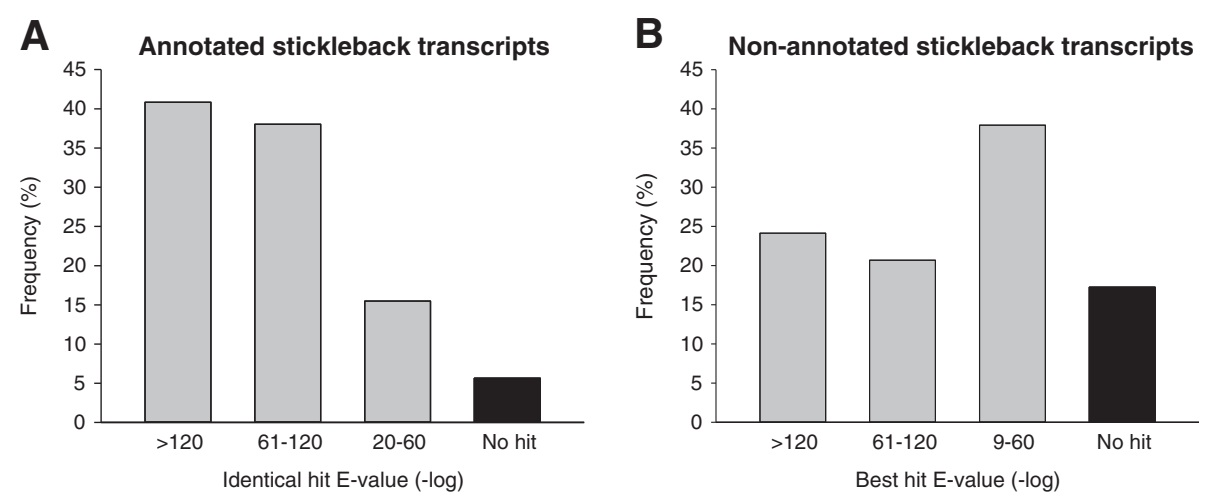

Figure 3 Quality control of the gilthead sea bream transcriptome annotation. Frequency distribution of BlastX hit results for annotated (A) and non-annotated (B) stickleback transcripts.

intended to become a dynamic and useful tool for scientific community. With this interface, data can be queried using different strategies, such as several Blast options or direct word search for annotation or GO terms. Search results provide additional information for each sequence, like its length, depth or the related Swissprot accession for the assigned annotation, among others.

\section{Conclusions}

A gilthead sea bream transcriptome database has been constructed by de novo assembly of five normalized 454 libraries from metabolically and immunologically relevant tissues combined with public sequences that included nine SSH libraries. This approach yielded 125,263 different sequences, and for 63,880 a reliable annotation was found, resulting in 21,384 different gene descriptions that comprised a vast array of functional categories and biological pathways. This constitutes the largest and most complete transcriptome reported to date for gilthead sea bream, having a size and depth equivalent to those reported in the Ensembl genome database for Atlantic cod and other cultured fish species. The information of annotated contigs has been semi-automatically corrected and filtered for redundancies, and is stored in a web database (http://www.nutrigroup-iats.org/seabreamdb) that has been provided with Blast and other search options for the scientific community. This represents a valuable tool to assist fish phenotyping and the concomitant development of molecular biomarker panels (microarrays or process-focused PCR-arrays) of prognostic and diagnostic value to cope with developmental-, nutritional-, environmental- and disease-related stressors.

\section{Methods}

Experimental setup and tissue sampling

Juveniles of gilthead sea bream were maintained under intensive rearing conditions in the indoor experimental facilities of the Institute of Aquaculture Torre de la Sal

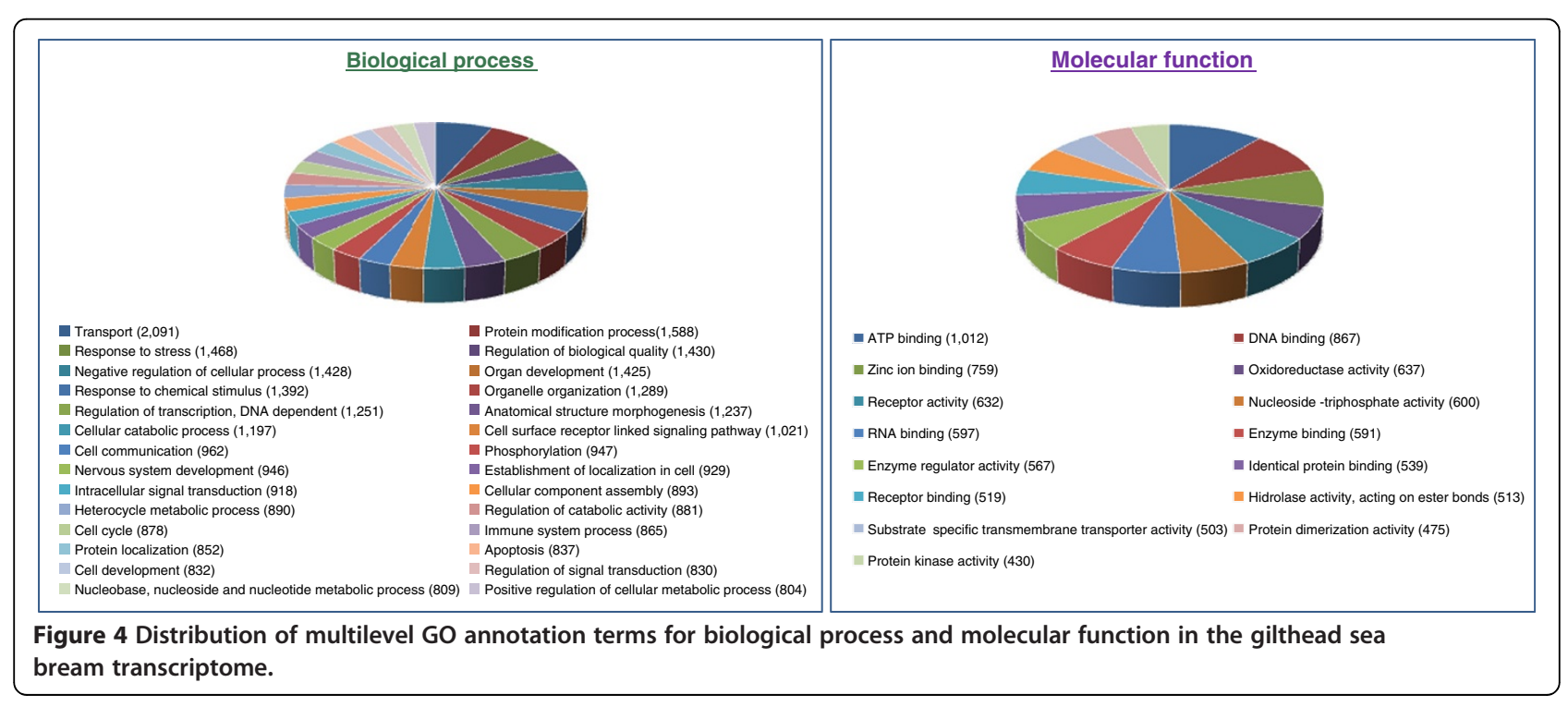


Table 3 Most significant canonical pathways determined by Ingenuity Pathway Analysis and represented in the assembled gilthead sea bream transcriptome

\begin{tabular}{|c|c|c|c|c|c|}
\hline Canonical pathway & E-value & Molecules & Canonical pathway & E-value & Molecules \\
\hline Regulation of elF4 and p70S6K Signaling & $1.58 \mathrm{E}-23$ & 130 & Erythropoietin Signaling & $8.32 \mathrm{E}-08$ & 56 \\
\hline Purine Metabolism & $7.94 \mathrm{E}-23$ & 219 & Ubiquinone Biosynthesis & $9.12 \mathrm{E}-08$ & 58 \\
\hline EIF2 Signaling & $3.98 \mathrm{E}-21$ & 156 & AMPK Signaling & $9.33 \mathrm{E}-08$ & 100 \\
\hline Protein Ubiquitination Pathway & $7.94 \mathrm{E}-20$ & 207 & Induction of Apoptosis by HIV1 & $1.05 \mathrm{E}-07$ & 51 \\
\hline Glucocorticoid Receptor Signaling & $2.51 \mathrm{E}-17$ & 203 & IL-2 Signaling & 1,17E-07 & 46 \\
\hline mTOR Signaling & $2.51 \mathrm{E}-16$ & 148 & CD40 Signaling & $1,20 \mathrm{E}-07$ & 53 \\
\hline Molecular Mechanisms of Cancer & $6.31 \mathrm{E}-16$ & 250 & Role of NFAT in Cardiac Hypertrophy & $1,29 \mathrm{E}-07$ & 131 \\
\hline Mitochondrial Dysfunction & $1.00 \mathrm{E}-15$ & 114 & HGF Signaling & $1,48 \mathrm{E}-07$ & 77 \\
\hline Integrin Signaling & $6.31 \mathrm{E}-14$ & 155 & FLT3 Signaling in Hematopoietics & $1,55 \mathrm{E}-07$ & 59 \\
\hline Inositol Phosphate Metabolism & $2.51 \mathrm{E}-13$ & 122 & Progenitor Cells & & \\
\hline Thrombin Signaling & $1.58 \mathrm{E}-12$ & 147 & Fcy Receptor-mediated Phagocytosis in & $1,58 \mathrm{E}-07$ & 73 \\
\hline Signaling by Rho Family GTPases & $1.58 \mathrm{E}-12$ & 174 & Macrophages and Monocytes & & \\
\hline Estrogen Receptor Signaling & $3.16 \mathrm{E}-12$ & 104 & Sphingosine-1-phosphate Signaling & $1,66 \mathrm{E}-07$ & 86 \\
\hline CXCR4 Signaling & $5.01 \mathrm{E}-12$ & 121 & FAK Signaling & $1,66 \mathrm{E}-07$ & 69 \\
\hline Oxidative Phosphorylation & $6.31 \mathrm{E}-12$ & 115 & Paxillin Signaling & $2,09 \mathrm{E}-07$ & 78 \\
\hline Acute Phase Response Signaling & $7.94 \mathrm{E}-12$ & 130 & Small Cell Lung Cancer Signaling & $2,40 \mathrm{E}-07$ & 58 \\
\hline Breast Cancer Regulation by Stathmin 1 & $1.26 \mathrm{E}-11$ & 147 & T Cell Receptor Signaling & $3,31 \mathrm{E}-07$ & 75 \\
\hline Type II Diabetes Mellitus Signaling & $3.98 \mathrm{E}-11$ & 94 & B Cell Receptor Signaling & $3,31 \mathrm{E}-07$ & 105 \\
\hline Huntington's Disease Signaling & $5.01 \mathrm{E}-11$ & 161 & Production of Nitric Oxide and Reactive & $3,72 \mathrm{E}-07$ & 126 \\
\hline Pyrimidine Metabolism & $7.94 \mathrm{E}-11$ & 111 & Oxygen Species in Macrophages & & \\
\hline NGF Signaling & $7.94 \mathrm{E}-11$ & 86 & LPS-stimulated MAPK Signaling & $3,89 \mathrm{E}-07$ & 59 \\
\hline PI3K/AKT Signaling & $1.10 \mathrm{E}-10$ & 96 & Insulin Receptor Signaling & $3,98 \mathrm{E}-07$ & 96 \\
\hline RAR Activation & $1.38 \mathrm{E}-10$ & 130 & Cyclins and Cell Cycle Regulation & $3,98 \mathrm{E}-07$ & 63 \\
\hline Androgen Signaling & $1.62 \mathrm{E}-10$ & 91 & PI3K Signaling in B Lymphocytes & 4,57E-07 & 99 \\
\hline Tight Junction Signaling & $1.86 \mathrm{E}-10$ & 120 & IGF-1 Signaling & $4,68 \mathrm{E}-07$ & 76 \\
\hline Chronic Myeloid Leukemia Signaling & $4.68 \mathrm{E}-10$ & 78 & p53 Signaling & $5,25 \mathrm{E}-07$ & 72 \\
\hline NRF2-mediated Oxidative Stress Response & $8.51 \mathrm{E}-10$ & 134 & VEGF Signaling & $5,62 \mathrm{E}-07$ & 68 \\
\hline PPARa/RXRa Activation & $1.12 \mathrm{E}-09$ & 128 & Prostate Cancer Signaling & $6,03 \mathrm{E}-07$ & 64 \\
\hline Actin Cytoskeleton Signaling & $1.78 \mathrm{E}-09$ & 154 & Nicotinate and Nicotinamide Metabolism & $6,46 \mathrm{E}-07$ & 77 \\
\hline Ceramide Signaling & $2.00 \mathrm{E}-09$ & 66 & Relaxin Signaling & $6,46 \mathrm{E}-07$ & 98 \\
\hline Role of Tissue Factor in Cancer & 2.29E-09 & 87 & Glioma Signaling & $7,24 \mathrm{E}-07$ & 73 \\
\hline Hereditary Breast Cancer Signaling & 3.31E-09 & 92 & Pentose Phosphate Pathway & $7,94 \mathrm{E}-07$ & 29 \\
\hline ERK/MAPK Signaling & $3.72 \mathrm{E}-09$ & 135 & Pancreatic Adenocarcinoma Signaling & $8,13 \mathrm{E}-07$ & 80 \\
\hline NF-KB Activation by Viruses & 4.57E-09 & 62 & Mitotic Roles of Polo-Like Kinase & $8,51 \mathrm{E}-07$ & 51 \\
\hline Apoptosis Signaling & 4.68E-09 & 73 & Acute Myeloid Leukemia Signaling & 9,33E-07 & 61 \\
\hline Ga12/13 Signaling & 4.90E-09 & 93 & PDGF Signaling & $9,33 \mathrm{E}-07$ & 57 \\
\hline Cardiac Hypertrophy Signaling & $5.13 \mathrm{E}-09$ & 159 & CNTF Signaling & $1,05 \mathrm{E}-06$ & 44 \\
\hline Rac Signaling & 5.37E-09 & 83 & Endometrial Cancer Signaling & $1,05 \mathrm{E}-06$ & 44 \\
\hline Germ Cell-Sertoli Cell Junction Signaling & $6.17 \mathrm{E}-09$ & 118 & Thrombopoietin Signaling & 1,17E-06 & 46 \\
\hline ILK Signaling & $6.76 \mathrm{E}-09$ & 135 & Melanocyte Development and Pigmentation & $1,29 \mathrm{E}-06$ & 66 \\
\hline Renin-Angiotensin Signaling & 7.94E-09 & 86 & Signaling & & \\
\hline Protein Kinase A Signaling & $8.51 \mathrm{E}-09$ & 212 & Prolactin Signaling & $1,45 \mathrm{E}-06$ & 58 \\
\hline Aldosterone Signaling in Epithelial Cells & 8.91E-09 & 117 & Colorectal Cancer Metastasis Signaling & $1,58 \mathrm{E}-06$ & 161 \\
\hline
\end{tabular}


Table 3 Most significant canonical pathways determined by Ingenuity Pathway Analysis and represented in the assembled gilthead sea bream transcriptome (Continued)

\begin{tabular}{|c|c|c|c|c|c|}
\hline RhoGDI Signaling & $9.12 \mathrm{E}-09$ & 129 & RANK Signaling in Osteoclasts & $1,82 \mathrm{E}-06$ & 67 \\
\hline p70S6K Signaling & $1.00 \mathrm{E}-08$ & 95 & IL-17A Signaling in Airway Cells & $2,19 \mathrm{E}-06$ & 51 \\
\hline IL-8 Signaling & $1.02 \mathrm{E}-08$ & 127 & Aminoacyl-tRNA Biosynthesis & $2,40 \mathrm{E}-06$ & 30 \\
\hline IL-15 Signaling & $1.15 \mathrm{E}-08$ & 53 & G Beta Gamma Signaling & 2,51E-06 & 68 \\
\hline fMLP Signaling in Neutrophils & $1.23 \mathrm{E}-08$ & 85 & Telomerase Signaling & 2,69E-06 & 75 \\
\hline Phospholipase C Signaling & $1.78 \mathrm{E}-08$ & 163 & IL-6 Signaling & $3,16 \mathrm{E}-06$ & 71 \\
\hline PAK Signaling & $1.91 \mathrm{E}-08$ & 72 & Role of BRCA1 in DNA Damage Response & $3,24 \mathrm{E}-06$ & 46 \\
\hline P2Y Purigenic Receptor Signaling Pathway & $2.24 \mathrm{E}-08$ & 93 & Death Receptor Signaling & $3,39 \mathrm{E}-06$ & 48 \\
\hline HMGB1 Signaling & $3.02 \mathrm{E}-08$ & 75 & IL-3 Signaling & $3,39 \mathrm{E}-06$ & 56 \\
\hline Leukocyte Extravasation Signaling & $3.16 \mathrm{E}-08$ & 137 & Starch and Sucrose Metabolism & $3,47 \mathrm{E}-06$ & 54 \\
\hline RhoA Signaling & $3.89 \mathrm{E}-08$ & 86 & GM-CSF Signaling & $3,47 \mathrm{E}-06$ & 50 \\
\hline PTEN Signaling & 4.07E-08 & 84 & Angiopoietin Signaling & 3,47E-06 & 52 \\
\hline Virus Entry via Endocytic Pathways & $4.57 \mathrm{E}-08$ & 72 & Non-Small Cell Lung Cancer Signaling & $3,47 \mathrm{E}-06$ & 52 \\
\hline Growth Hormone Signaling & $5.13 \mathrm{E}-08$ & 57 & Role of NFAT in Regulation of the Immune & $3,72 \mathrm{E}-06$ & 120 \\
\hline JAK/Stat Signaling & $6.31 \mathrm{E}-08$ & 52 & Response & & \\
\hline Lymphotoxin $\beta$ Receptor Signaling & $7.08 \mathrm{E}-08$ & 47 & Neuregulin Signaling & $3,72 \mathrm{E}-06$ & 67 \\
\hline
\end{tabular}

(IATS), following standard conditions of photoperiod and temperature at our latitude $\left(40^{\circ} 5^{\prime} \mathrm{N}, 0^{\circ} 10^{\prime} \mathrm{E}\right)$. Animals were fed with conventional diets and culture densities remained lower than $15 \mathrm{Kg} / \mathrm{m}^{3}$. Naïve stock fish were sampled after overnight fasting for blood, skeletal muscle and intestine. Blood was taken from the caudal vein with EDTA-treated syringes and $150 \mu \mathrm{l}$ were disposed in cooled eppendorf tubes with $500 \mu \mathrm{l}$ of lysis solution until RNA extraction (Real Total RNA Spin Blood Kit, Durviz). Prior to tissue collection, fish were killed by cervical section. Skeletal muscle and intestine samples were then rapidly excised, frozen in liquid nitrogen and stored at $-80^{\circ} \mathrm{C}$ until RNA extraction. Additionally, another stock of fish was infected with the myxosporean parasite Enteromyxum leei by exposure to contaminated effluent, following the infection procedure previously described [32], and samples of head kidney, the equivalent to mammalian bone marrow, were taken after 38 (HK-38, early immune response) and 105 days (HK-105, chronic immune response) post exposure. All procedures were carried out according to the national and institutional regulations on animal experimental handling (IATS-CSIC Review Board).

\section{RNA extraction}

Total RNA from each individual tissue sample was isolated by means of the Ambion MagMax-96 for Microarray kit (Applied Biosystems) after tissue homogenization in TRI reagent at a concentration of $100 \mathrm{mg} / \mathrm{ml}$ following the manufacturers' instructions. Purification of total RNA from fish blood was performed according to the procedure of Real Total RNA Spin Blood Kit (Durviz). RNA quantity and purity was determined by Nanodrop (Thermo Scientific) and Agilent 2100 bioanalyzer (Agilent Technologies). Tissue samples of higher quality (absorbance ratios at $260 \mathrm{~nm} / 280 \mathrm{~nm}$ above 1.9 and RNA integrity numbers between 9.2 and 10) were selected for high-throughput sequencing.

\section{CDNA synthesis and normalization}

For each tissue and condition (skeletal muscle, intestine, blood, HK-38, HK-105), a single RNA sample (700 ng) was taken for polyA cDNA synthesis using the MINT kit (Evrogen). To increase the rate recovery of rare and unique transcripts, amplified cDNAs were normalized by duplex-specific nuclease with the Trimmer kit (Evrogen) [33] following manufacturer's indications. Normalized cDNAs samples were measured with QuantiT PicoGreen dsDNA quantification Kit (Life Technologies) using a VersaFluorTM Fluorometer system (Bio-Rad).

\section{Libraries construction, pyrosequencing and assembly}

For each normalized cDNA, $500 \mathrm{ng}$ were sheared into small fragments (250-600 nt) by nebulization with compressed nitrogen. Then, sequencing adapters were ligated to the blunt ends of fragments, and an emulsion PCR (emPCR) was performed. After emPCR, beads with the cloned amplicons were enriched, loaded onto the 454 microtiter plate and sequenced with a Titanium GS FLX 454 platform (Roche). The sff files containing all reads for each library have been deposited to NCBI Short Read Archive under accession ERP001436.

The quality of the reads was assessed with PERL scripts developed at Lifesequencing S.L. (Valencia, Spain) for 
trimming of adaptors and validation of high quality sequences. Only high-quality reads (q-value > 25) passed the filter for further assembly and they were assembled using Newbler version 2.5.3 program (454 Life Science-Roche) with the parameters by default with the cDNA option.

\section{De novo assembly and annotation}

According to the presented scheme pipeline (Figure 1), the set of nucleotide sequences for de novo gilthead sea bream transcriptome assembly was composed of i) unassembled high-quality reads from five 454 libraries (2,945.914 reads), ii) available mRNA sequences from GenBank database (1,733 sequences) and iii) EST collections made available by the Consortium of Marine Genomics Europe [34] and the AQUAFIRST [1] and AQUAMAX [3] EU projects (80,956 ESTs). All sequences were edited to remove vector and adaptor sequences, and cleaned and filtered before assembly and annotation by the SIGENAE information system (INRA Toulouse, France). Cleaning involved masking of poor quality bases and low complexity sequences such as polyA tails. Filtering removed contaminating sequences (bacteria, yeast) and only high quality sequences with more than 100 bases in length were retained. The global assembly was performed by means of the MIRA software version 3.2.0 [35,36].

Assembled contigs and singletons were annotated searching sequence homologies against following databases: UniProtKB/Swiss-Prot, UniProtKB/TrEMBL, RefSeq Protein, Pfam, RefSeq RNA Index Blast, TIGR Fugu, TIGR Medaka, TIGR Salmon, TIGR Trout, TIGR ZebraFish, UniGene FatheadMinnow, UniGene Fugu, UniGene Human, UniGene KilliFish, UniGene Medaka, UniGene Salmon, UniGene Trout, UniGene ZebraFish, Ensembl Fugu transcripts, Ensembl Human transcripts, Ensembl Medaka transcripts, Ensembl Tetraodon transcripts and Ensembl ZebraFish transcripts. The e-value threshold value to determine similarities was set to $1 \mathrm{e}-5$, and the Uniprot entry to which they received the highest similarity was usually assigned as the gene identity.

\section{Database quality control}

Transcriptome sequences of the fish three-spined stickleback (Gasterosteus aculeatus) were retrieved from the Ensembl genome database (http://www.ensembl.org/ Gasterosteus_aculeatus). Then, randomly selected stickleback sequences $(n=200)$ were compared in a similarity search by BlastX (E-value < 1e-9) into the newly developed gilthead sea bream assembled transcriptome database. For annotated stickleback transcripts, hit results were only considered positive when the most similar gilthead sea bream sequence shared the same annotation.
Automated frameshift correction and redundancy filtering To correct the 454 sequencing errors due to frameshifting at homopolymer regions [28], a pipeline based on the combination of the NCBI-Blast package [37] with HMMER [http://hmmer.janelia.org], ClustalW [38], HMM-FRAME [39], PFAM [40] and GyDB [41] databases of HMMs was designed. Additional file 3 implements a most extensive detail of this pipeline and the corrections (punctual insertions/deletions) performed on each sequence.

GPRO 1.1 software [42] was employed to select the most representative sequences among the distinct annotations for a transcribed gene using the algorithm 1, which is a normalized combination of the most relevant Blast statistics with the assembly depth of the sequences:

$$
\begin{aligned}
\text { Algorithm } 1= & \frac{\text { HSPquery }}{100} \times \frac{\text { HSPhit }}{100} \times \frac{\text { Similarity }}{100} \\
& \times \frac{1}{E-\text { value }} \times \text { Depth }
\end{aligned}
$$

For more extensive information about the statistics composing the algorithm please refer the Blast user guide at NCBI [http://www.ncbi.nlm.nih.gov/books/ NBK1763/]. The software reads in csv format the annotation file of the whole transcriptome and then lets to state one or more classificatory filters to run the algorithm. When various sequences shared the same SwissProt description and a nucleotide identity higher than 95\%, they were grouped in the same cluster, retaining within the same categorization the sequences covering non-overlapping regions of the mapped Refseq protein or complete sequences sharing the same description.

\section{Functional analysis}

Gene ontology annotation was made from the nucleotide sequences of the most representative contig/singleton for each gene identity by means of the Blast2GO software [43] with a threshold cutoff set at 1e-3. Pathway analysis of annotated sequences was performed using the IPA software (http://www.ingenuity.com). The dynamic canonical pathways contained in IPA are wellcharacterized metabolic and cell-signaling pathways that come from specific journal articles, review articles, textbooks and the Kyoto encyclopedia of genes and genomes (KEGG). The IPA canonical pathways display genes/proteins involved, their interactions and the cellular and metabolic reactions in which the pathway is involved. To provide analysis, IPA must be supplied with the Uniprot accession of genes belonging to one of the following model species: Human (Homo sapiens), house mouse (Mus musculus), rat (Rattus norvegicus), fruit fly (Drosophila melanogaster), thale cress (Arabidopsis thaliana), the nematode Caenorhabditis elegans, the bacteria Escherichia coli or the yeast Saccharomyces cerevisiae. Hence, for each annotated sea bream 
sequence the protein equivalence for one of the three higher vertebrates IPA model species was searched in Uniprot, and the corresponding accession number was included in the analysis.

\section{Statistics}

Fisher's exact test was used in IPA analysis to estimate the significance of the incidence of different canonical pathways. This method calculates the probability that the association between experimental gene set and the reference gene set associated with a canonical pathway is due to random chance. A P-value $\leq 0.05$ was considered statistically significant and indicated a nonrandom enrichment of an experimental dataset by members of a specific pathway.

\section{Additional files}

\section{Additional file 1: Complete list of IPA canonical pathways, with the} ratio of gilthead sea bream sequences of the reported transcriptome detected for each one.

Additional file 2: Biological networks of genes linked to the canonical pathways "mTOR Signaling" (A), "Mitochondrial Dysfunction" (B), "Tight Junction Signaling" (C), "B Cell Receptor Signaling" (D), "Acute phase response signaling" (E) and "Complement System" (F) according to Ingenuity Pathway Analysis. Genes that are present in the gilthead sea bream nucleotide database are shown with grey shading. Direct interactions are shown as solid lines and indirect as dashed lines.

Additional file 3: Post-processing data and pipeline for frameshift correction. Zip file contains a Word document with an explanation of the distinct steps of the frameshifts processing pipeline accompanied by a graphical description of the pipeline schematics, and an Excel document reporting the frameshift corrections.

\section{Competing interests}

The authors declare that they have no competing interests.

\section{Authors' contributions}

JPS, ASB and JACG conceived and designed the study. IE and ASB performed the parasite challenges. ABN, LBP and GBL maintained and sampled stock fish. JACG and IE performed RNA extractions. JACG and JPS conducted statistical, gene ontology and pathway analysis of data. ABM and LBP contributed to manual curation of the nucleotide database. JACG, ASB and JPS wrote the manuscript. All authors read, critically revised and approved the final manuscript.

\section{Acknowledgements}

This work was funded under EU seventh Framework Programme by projects ARRAINA (Advanced Research Initiatives for Nutrition \& Aquaculture, FP7/ 2007-2013; grant agreement no 288925) and AQUAEXCEL (Aquaculture Infrastructures for Excellence in European Fish Research, FP7/2007-2012; grant agreement no 262336). The views expressed in this work are the sole responsibility of the authors and do not necessarily reflect the views of the European Commission. Additional funding was obtained by Generalitat Valenciana (research grant PROMETEO 2010/006) and Spanish Government through AQUAGENOMICS (Ingenio-2010 Programme), AQUAFAT (AGL200907797) and ENTEROMYXCONTROL (AGL2009-13282-C02-01) projects.

\section{Author details}

${ }^{1}$ Nutrigenomics and Fish Growth Endocrinology Group, Department of Marine Species Biology, Culture and Pathology, Institute of Aquaculture Torre de la Sal, Castellón, CSIC, Spain. ${ }^{2}$ Fish Pathology Group, Department of Marine Species Biology, Culture and Pathology. Institute of Aquaculture Torre de la Sal, Castellón, CSIC, Spain.
Received: 29 November 2012 Accepted: 8 March 2013

Published: 15 March 2013

\section{References}

1. Calduch-Giner JA, Davey G, Saera-Vila A, Houeix B, Talbot A, Prunet P, Cairns MT, Pérez-Sánchez J: Use of microarray technology to assess the time course of liver stress response after confinement exposure in gilthead sea bream (Sparus aurata L.). BMC Genomics 2010, 11:193.

2. Davey GC, Calduch-Giner JA, Houeix B, Talbot A, Sitjà-Bobadilla A, Prunet P, Pérez-Sánchez J, Cairns MT: Molecular profiling of the gilthead sea bream (Sparus aurata L.) response to chronic exposure to the myxosporean parasite Enteromyxum leei. Mol Immunol 2011, 48:2102-2112.

3. Calduch-Giner JA, Sitjà-Bobadilla A, Davey GC, Cairns MT, Kaushik S, Pérez-Sánchez J: Dietary vegetable oils do not alter the intestine transcriptome of gilthead sea bream (Sparus aurata), but modulate the transcriptomic response to infection with Enteromyxum leei. BMC Genomics 2012, 13:470.

4. Ferraresso S, Vitulo N, Mininni AN, Romualdi C, Cardazzo B, Negrisolo E, Reinhardt R, Canario AVM, Patarnello T, Bargelloni L: Development and validation of a gene expression oligo microarray for the gilthead sea bream (Sparus aurata). BMC Genomics 2008, 9:580.

5. Sarropoulou E, Kotoulas G, Power DM, Geisler R: Gene expression profiling of gilthead sea bream during early development and detection of stress-related genes by the application of CDNA microarray technology. Physiol Genomics 2005, 23:182-191.

6. Teles M, Boltaña S, Reyes-López F, Santos MA, Mackenzie S, Tort L: Effects of chronic cortisol administration of GR and the liver transcriptome in Sparus aurata. Mar Biotechnol 2013, 15:104-114.

7. Vieira FA, Gregório SF, Ferraresso S, Thorne MAS, Costa R, Milan M, Bargelloni L, Clark MS, Canario AVM, Power DM: Skin healing and scale regeneration in fed and unfed sea bream Sparus auratus. BMC Genomics 2011, 12:490.

8. Adzhubei AA, Vlasova AV, Hagen-Larsen H, Ruden TA, Laerdahl JK, Hoyheim B Annotated expressed sequence tags (ESTs) from pre-smolt Atlantic salmon (Salmo salar) in a searchable data resource. BMC Genomics 2007, 8:209.

9. Gonzalez S, Chatziandreou N, Nielsen M, Li W, Rogers J, Taylor R, Santos Y, Cossins A: Cutaneous immune responses in the common carp detected using transcript analysis. Mol Immunol 2007, 44:1675-1690.

10. Lee BY, Howe AE, Conte MA, D'Cotta H, Pepey E, Baroiller JF, di Palma F, Carleton KL, Kocher TD: An EST resource for tilapia based on 17 normalized libraries and assembly of 116,899 sequence tags. BMC Genomics 2010, 11:278.

11. Pardo BG, Fernández C, Millán A, Bouza C, Vázquez-López A, Vera M, Alvarez-Dios JA, Calaza M, Gómez-Tato A, Vázquez M, Cabaleiros S, Magariños B, Lemos ML, Leiro JM, Martínez P: Expressed sequence tags (ESTs) from immune tissues of turbot (Scophthalmus maximus) challenged by pathogens. BMC Vet Res 2008, 4:37.

12. Ansorge WJ: Next-generation DNA sequencing techniques. New Biotechnology 2009, 25:195-203

13. Salem M, Rexroad CE III, Wang J, Thorgaard GH, Yao J: Characterization of the rainbow trout transcriptome using Sanger and 454-pyrosequencing approaches. BMC Genomics 2010, 11:564.

14. Le Cam A, Bobe J, Bouchez O, Cabau C, Kah O, Klopp C, Lareyre JJ, Le Guen I, Lluch J, Montfort J, Moreews F, Nicol B, Prunet P, Rescan PY, Servili A, Guiguen Y: Characterization of rainbow trout gonad, brain and gill deep cDNA repertoires using a Roche 454-Titanium sequencing approach. Gene 2012, 500:32-39.

15. Johansen SD, Karlsen BO, Furmanek T, Andreassen M, Je rgensen TE, Bizuayehu TT, Breines R, Emblem Å, Kettunen P, Luukko K, Edvardsen RB, Nordeide JT, Coucheron DH, Moum T: RNA deep sequencing of the Atlantic cod transcriptome. Comp Biochem Physiol part D 2011, 6:18-22.

16. Pereiro $P$, Balseiro $P$, Romero A, Dios $S$, Forn-Cuni G, Fuste B, Planas JV, Beltran S, Novoa B, Figueras A: High-throughput sequence analysis of turbot (Scophthalmus maximus) transcriptome using 454-pyrosequencing for the discovery of antiviral immune genes. PLoS One 2012, 7:e35369.

17. Yúfera M, Halm S, Beltran S, Fusté B, Planas JV, Martínez-Rodríguez G: Transcriptomic characterization of the larval stage in gilthead seabream (Sparus aurata) by 454 pyrosequencing. Mar Biotechnol 2012, 14:423-435.

18. Garcia de la Serrana Castillo D, Estevez A, Andree K, Johnston IA: Fast skeletal muscle transcriptome of the gilthead sea bream (Sparus aurata) determined by next generation sequencing. BMC Genomics 2012, 13:181. 
19. Meyer E, Aglyamova GA, Wang S, Buchanan-Carter J, Abrego D, Colbourne JK, Willis BL, Matz MV: Sequencing and de novo analysis of a coral larval transcriptome using 454 GSFIx. BMC Genomics 2009, 10:219.

20. Gao Z, Luo W, Liu H, Zeng C, Liu X, Yi S, Wang W: Transcriptome analysis and SSR/SNP markers information of the blunt snout bream (Megalobrama amblycephala). PLoS One 2012, 7:e42637.

21. Fu B, He S: Transcriptome analysis of silver carp (Hypophthalmichthys molitrix) by paired-end DNA sequencing. DNA Res 2012, 19:131-142.

22. Roest Crollius H, Weissenbach J: Fish genomics and biology. Genome Res 2005, 15:1675-1682.

23. Sato $Y$, Hashiguchi $Y$, Nishida M: Temporal pattern of loss/persistence of duplicate genes involved in signal transduction and metabolic pathways after teleost-specific genome duplication. BMC Evol Biol 2009, 9:127.

24. Mattick JS: The central role of RNA in human development and cognition. FEBS Lett 2011, 585:1600-1616.

25. Kapranov P, St Laurent G: Dark matter RNA: existence, function, and controversy. Front Genet 2012, 3:57.

26. Huse SM, Huber JA, Morrison HG, Sogin ML, Welch DM: Accuracy and quality of massively parallel DNA pyrosequencing. Genome Bio/ 2007 8:R143.

27. Balzer S, Malde K, Lanzén A, Sharma A, Jonassen I: Characteristics of 454 pyrosequencing data - enabling realistic simulation with flowsim. Bioinformatics 2010, 26:i420-i425.

28. Loman N, Misra RV, Dallman TJ, Constantinidou C, Gharbia SE, Wain J, Pallen MJ: Performance comparison of benchtop high-throughput sequencing platforms. Nat Biotechnol 2012, 30:434-439.

29. Van Herwaarden $A E$, Van Waterschoot $R A B$, Schinkel $A H$ : How important is intestinal cytochrome P450 3A metabolism? Trends Pharmacol Sci 2009, 30:223-227

30. Khatri P, Sirota M, Butte AJ: Ten years of pathway analysis: current approaches and outstanding challenges. PLoS Comput Biol 2012, 8:e1002375.

31. Li S, Pozhitkov A, Ryan RA, Manning CS, Brown-Peterson N, Brouwer M: Constructing a fish metabolic network model. Genome Biol 2010, 11:R115.

32. Sitjà-Bobadilla A, Diamant A, Palenzuela O, Alvarez-Pellitero P: Effect of host factors and experimental conditions on the horizontal transmission of Enteromyxum leei (Myxozoa) to gilthead sea bream (Sparus aurata L.) and European sea bass (Dicentrarchus labrax L.). J Fish Dis 2007, 30:243-250

33. Zhulidov PA, Bogdanova EA, Shcheglov AS, Vagner LL, Khaspekov GL, Kozhemyako VB, Matz MV, Meleshkevitch E, Moroz LL, Lukyanov SA, Shagin DA: Simple cDNA normalization using kamchatka crab duplex-specific nuclease. Nucleic Acids Res 2004, 32:e37.

34. Louro B, Passos ALS, Souche EL, Tsigenopoulos C, Beck A, Lagnel J, Bonhomme F, Cancela L, Cerdà J, Clark M, Lubzens E, Magoulas A, Planas JV, Volckaert FAM, Reinhardt R, Canario AVM: Gilthead sea bream (Sparus auratus) and European sea bass (Dicentrarchus labrax) expressed sequence tags: Characterization, tissue-specific expression and gene markers. Marine Genomics 2010, 3:179-191.

35. Chevreux B, Wetter $T$, Suhai $S$ : Genome sequence assembly using trace signals and additional sequence information computer science and biology. Proceedings of the German Conference on Bioinformatics 1999:45-56.

36. Chevreux B, Pfisterer T, Drescher B, Driesel AJ, Müller WE, Wetter T, Suhai S: Using the miraEST Assembler for Reliable and Automated mRNA Transcript Assembly and SNP Detection in Sequenced ESTs. Genome Res 2004, 14:1147-1159.

37. Altschul SF, Madden TL, Schaffer AA, Zhang J, Zhang Z, Miller W, Lipman DJ: Gapped BLAST and PSI-BLAST: a new generation of protein database search programs. Nucleic Acids Res 1997, 25:3389-3402.

38. Thompson JD, Gibson TJ, Higgins DG: Multiple sequence alignment using ClustalW and ClustalX. Curr Protoc Bioinformatics 2002, 23:2.3.1-2.3.22.

39. Zhang $Y$, Sun $Y$ : HMM-FRAME: accurate protein domain classification for metagenomic sequences containing frameshift errors. BMC Bioinformatics 2011, 12:198.

40. Punta M, Coggill PC, Eberhardt RY, Mistry J, Tate J, Boursnell C, Pang N, Forslund K, Ceric G, Clements J, Heger A, Holm L, Sonnhammer ELL, Eddy SR, Bateman A, Finn RD: The Pfam protein families database. Nucleic Acids Res 2012, 40:D290-D301.

41. Llorens C, Futami R, Covelli L, Dominguez-Escriba L, Viu JM, Tamarit D, Aguilar-Rodríguez J, Vicente-Ripolles M, Fuster G, Bernet GP, Maumus F, Munoz-Pomer A, Sempere JM, Latorre A, Moya A: The Gypsy Database
(GyDB) of mobile genetic elements: release 2.0. Nucleic Acids Res 2011, 39:D70-D74.

42. Futami R, Muñoz-Pomer L, Viu JM, Dominguez-Escriba L, Covelli L, Bernet GP, Sempere JM, Moya A, Llorens C: GPRO The professional tool for annotation, management and functional analysis of omic databases. Biotechvana Bioinformatics 2011. SOFT3.

43. Conesa A, Götz S, García-Gómez JM, Perol J, Talón M, Robles M: Blast2GO: a universal tool for annotation, visualization and analysis in functional genomics research. Bioinformatics 2005, 21:3674-3676.

doi:10.1186/1471-2164-14-178

Cite this article as: Calduch-Giner et al:: Deep sequencing for de novo construction of a marine fish (Sparus aurata) transcriptome database with a large coverage of protein-coding transcripts. BMC Genomics 2013 14:178.

\section{Submit your next manuscript to BioMed Central and take full advantage of:}

- Convenient online submission

- Thorough peer review

- No space constraints or color figure charges

- Immediate publication on acceptance

- Inclusion in PubMed, CAS, Scopus and Google Scholar

- Research which is freely available for redistribution

Submit your manuscript at www.biomedcentral.com/submit
C) BioMed Central 